\title{
ANTENNA IMPEDANCE IN A PLASMA : PROBLEMS RELEVANT TO RADIO ASTRONOMY MEASUREMENTS FROM SPACE VEHICLES
}

\author{
by D. WALSH and F. T. HADDOCK
}

(Radio Astronomy Observatory, University of Michigan, Ann Arbor, Michigan, U. S. A.)

RÉsumé. - On présente une revue critique des théories et des observations du comportement d'une antenne dans un plasma qui sont importantes en radioastronomie. On insiste plus particulièrement sur les antennes électriquement courtes. On envisage 3 cas; plasma froid sans champ magnétique, plasma chaud avec champ magnétique et plasma chaud sans champ magnétique. On discute de nouveaux résultats sur le cas d'un plasma froid sans champ magné. tique qui sont en bon accord, même dans les détails avec la théorie au voisinage du cutoff de la propagation de l'onde magnétoionique extraordinaire.

ABstract. - A critical review is given of the theory and experimental observations of antenna impedance behaviour in a plasma, as related to radio astronomy. The emphasis is on electrically small antennas. Three simplified cases of plasma are considered, namely, cold plasma without magnetic field, cold plasma with magnetic field and warm plasma without magnetic field. Newly reduced experimental results are reported for the case of cold plasma without magnetic field, showing detailed agreement with theory in the neighbourhood of a cutof in the propagation of the extraordinary magnetoionic wave.

Резюме. - Представлен критический обзор теорий и наблюдений поведения антенны в плазме, которые имеют большое значение дия радиоастрономии. С особои настоятельностью - электрически коротких антенн. Рассмотрены 3 случая : холодная плазма без магнитного поля, холодная плазма с магнитным полем и горячая плазма без магнитного поля. Новые результаты, которые находятся в хорошем согласии даже в подробностях с теорией около порогового распространения необыкновенной магнитоионной волны, обсуждены на случае холодной плазмы без магнитного поля.

\section{INTKODUCTION.}

Most radio astronomy measurements from space vehicles have been made or are planned at altitudes where observations may be affected by the ionized layers of the earth's outer atmosphere. Understanding of the behaviour of antenna impedance in a plasma is essential for correct interpretation of many such observations. The purpose of this paper is to review critically the current status of the relevant theory and related observations.

Frequent reference will be made to the equivalent circuit of figure 1. The antenna impedance $\mathrm{Z}_{\mathrm{A}}$ is represented by series resistance $\mathrm{R}_{\mathrm{A}}$ and reactance $\mathbf{X}_{\mathbf{A}}$; in general both are functions of frequency. In the following it will be assumed the antenna is lossless, so $R_{A}$ is purely radiation resistance. If it is receiving a signal, this may be represented by an equivalent generator with the open-circuit voltage of the antenna, $V_{A}$. When

$\left({ }^{*}\right)$ Work supported by NASA on contract Nasw-54 and grant NSG 181. the antenna is terminated by a load impedance $Z_{L}$, the voltage $V_{L}$ at the antenna terminals is related to $V_{A}$ by the relative impedances $Z_{A}$ and $Z_{L}$. $Z_{L}$ includes such quantities as shunt capacity inherent in the antenna mounting structure. $V_{L}$ is the quantity directly measured in practice, but it is desired to find $V_{A}$. If $Z_{L}$ is sufficiently large compared with $Z_{A}, V_{L}$ may be practically equal to $V_{A}$; otherwise knowledge of $Z_{A}$ is necessary to deduce $\mathrm{V}_{\mathrm{A}}$.

In a plasma, the impedance is a function of plasma frequency and static magnetic field. It will be convenient to introduce them by the para-

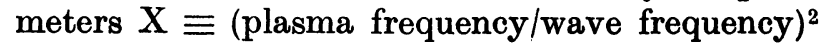
and $\mathrm{Y} \equiv$ (gyro frequency/wave frequency). The collisionless case only will be considered here. It is adequate for most problems in the topside ionosphere, and most of the results may be extended without difficulty to include collisions. The behavior of $R_{A}$ and $X_{A}$ as functions of $X$ and $Y$ has been the subject of considerable theoretical analysis.

When observing noise signals, it is convenient to 


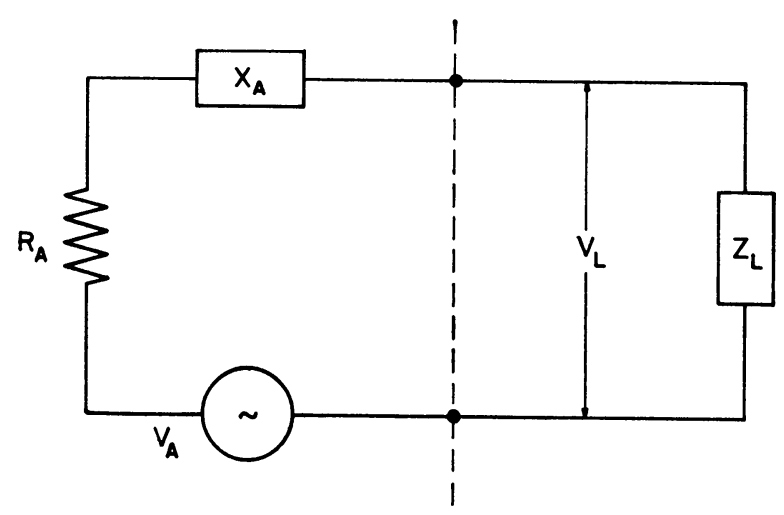

Fra. 1. - Equivalent circuit of terminated antenna. $\mathbf{Z}_{\mathbf{A}}=\mathbf{R}_{\mathbf{A}}+j \mathbf{X}_{\mathbf{A}}$ is the antenna impedance; $\mathbf{Z}_{\mathbf{L}}$ is the terminating impedance.

refer to antenna temperature, $T_{A}$, which is related to the mean square value of $V_{A}$ in a bandwith $B$ by

$$
\overline{\mathrm{V}_{\mathrm{A}}^{2}}=4 \mathrm{KT}_{\mathrm{A}} \mathrm{R}_{\mathrm{A}} \mathrm{B} .
$$

$T_{A}$ is the quantity ultimately desired. It represents an average radio brightness temperature for the region of sky observed. Given $\overline{\mathrm{V}_{\mathbf{A}}^{2}}$, knowledge of $R_{A}$ is necessary to obtain $T_{A}$.

An alternative approach for a short electric dipole is to express $V_{A}$ in terms of the effective length $h$ and the component of the incident electric field, $\mathbf{E}_{\text {tan }}$, which is tangential to the dipole,

$$
\overline{\mathrm{V}_{\mathbf{A}}^{2}}=h^{2} \overline{\mathrm{E}}_{\text {tan }}^{2} \text {. }
$$

For an elementary loop antenna, or magnetic dipole, the appropriate formulation is in terms of an effective area $A_{e}$ and the component of magnetic induction, $\mathrm{B}_{\text {norm}}$, which is normal to the plane of the loop (i. e. tangential to the magnetic dipole),

$$
\overline{\mathrm{V}_{\mathrm{A}}^{2}}=\omega^{2} \mathrm{~A}_{\epsilon}^{2} \overline{\mathrm{B}_{\text {norm }}^{2}} \text {. }
$$

Assuming the effective length or area is known, it is then necessary to relate the measured fields to the sky brightness. This has been discussed by HUarl [1] and by BuDDEN and HoGmL [2]. The formal equivalence of the theory involved in this topic with that involved in $\mathbf{R}_{\mathbf{A}}$ behavior in a plasma may be demonstrated. Either approach is valid in interpreting and discussing data. The impedance approach will be used hereafter.

Much of the discussion will deal with electrically small antennas, that is, those with all dimensions much less than any wavelength of interest. The emphasis will be on electric dipoles, though some reference to magnetic dipoles will be made. One advantage of antennas which are electrically small both in free space and in the plasma is that, to good approximation, the current distribution is the same in both cases. For a short electric dipole the current distribution is approximately " triangular" i. e. tapering linearly from the centre to the ends. For an electrically small loop, the current distribution is uniform. Many calculations are based on the assumption that the current distribution is unaffected by the plasma. If this is not true for any reason, the theoretical value of $\mathbf{R}_{\mathbf{A}}$ will be wrong. If this theoretical value is used to derive $T_{A}$, then the calculated $T_{A}$ will be wrong. This cannot be overcome by the approach of (1.2) or (1.3) since the effective length or area will be different from their values for the free-space current distribution, so the incident fields cannot be deduced correctly.

For a short electric dipole, $\mathbf{X}_{\mathbf{A}}$ is usually much greater than $\mathbf{R}_{\mathbf{A}}$. Consequently, though $\mathbf{X}_{\mathbf{A}}$ may be susceptible to direct measurement in practice, $R_{A}$ often is not.

In the steps outlined above for deriving $T_{A}$, knowledge of $\mathrm{X}_{A}$ alone is often adequate for deducing $V_{A}$ from $V_{L}$, but $R_{A}$ must be introduced to derive $T_{A}$ via (1.1). Thus while an accurate theory for $X_{A}$ in the plasma is not essential, one is essential for $\mathbf{R}_{\mathrm{A}}$. However, in addition to these requirements for analysis of data, there are other reasons for desiring reasonable theories both for $X_{A}$ and $R_{A}$. In order to make the above theoretical corrections, knowledge of the parameters $X$ and $Y$ is needed. The geomagnetic field is known well enough to give $Y$ adequately in most cases, but $\mathbf{X}$ must usually be derived from suitable measurements. Observations of $\mathbf{X}_{\mathbf{A}}$ and $\mathbf{R}_{\mathbf{A}}$ behavior may in fact give this information. Another reason for wishing to predict the behavior of $\mathbf{X}_{\mathbf{A}}$ and $\mathbf{R}_{\mathbf{A}}$ is to ensure that the operating ranges of observing instruments are correctly adjusted. Thus theories of both $R_{A}$ and $X_{A}$ in a plasma are desirable for reasons of data analysis, plasma diagnostics and equipment design.

It should be pointed out that the separation of the problems of $R_{A}$ and $X_{A}$ in a plasma results in considerable theoretical simplification. However, this is only justifiable for an electrically small antenna. The justification in this case may be seen by considering the fields of a Hertzian dipole. As shown in most texts, these fields contain terms proportional to $r^{-3}, r^{-2}$ and $r^{-1}$. The near field $r-3$ terms provide the dominant term in the impedance. They occur only in the electric field and, apart from the harmonic time variation, they are identical to the field of a static dipole. They 
account for the dominantly capacitive behavior of a short dipole in free space. This justifies a quasistatic approach to calculating the reactance of a short electric dipole in free space, either by solving Laplace's equation subject to the boundary conditions that the antenna surfaces are equipotentials (thus deriving the charge distribution on the antenna surface), or by solving Poisson's equation assuming a reasonable charge distribution. These methods have been applied in suitably modified form to antennas in plasmas. They are inherently incapable of generating the terms corresponding to the $r^{-1}$ fields in free space and are only valid provided the antenna is electrically small in the plasma. It is these latter fields which account for the radiated power and for $R_{A}$, so an alternative approach is necessary to derive $R_{A}$.

In the following sections, various approximations to antenna impedance in plasma will be examined. The behavior in a cold plasma without magnetic field, discussed first, is of value mainly in indicating trends which occur when a magnetic field is present, discussed second. The latter case is the one which has proved most important so far in analyzing observations. Finally, possible effects of " electroacoustic" fields, which can occur in a warm plasma due to pressure associated with thermal motions, are discussed. This subject is still somewhat speculative, but could turn out to be very significant. Ideally, the effects of a static magnetic field should be considered, but this would superpose complexity on speculation.

\section{Cold PLASMa Withodt Magnetic FIELD.}

The plasma behaves as a pure dielectric with refractive index $n=(1-\bar{X})^{1 / 2}$. For a small electric dipole it is readily shown that $R_{A}=n R_{A_{0}}$ and $\mathbf{X}_{\mathbf{A}}=n^{-2} \mathbf{X}_{\mathbf{A}_{0}}$. This $\mathbf{X}_{\mathbf{A}}$ behavior is correctly represented at all frequencies by an equivalent circuit in which the free space capacity $C_{0}$ is unchanged but is shunted by an inductance with a value which produces a parallel resonance at the plasma frequency. For a small magnetic dipole $\mathbf{R}_{\mathbf{A}}=n^{3} \mathbf{R}_{\mathbf{A}_{0}}$, but $\mathbf{X}_{\mathbf{A}}$ is unaffected by the plasma since the antenna is essentially an inductance.

As the local plasma frequency is increased, the mean square open circuit voltage $\overline{\mathrm{V}_{\mathbf{A}}^{2}}$ of a magnetic dipole will therefore fall more rapidly than that of an electric dipole by a factor $n^{2}$. However, as far as the signal $\mathrm{V}_{\mathrm{L}}^{2}$ at the receiver input terminals is concerned, the changing $\mathbf{X}_{\mathbf{A}}$ may act unfavorably against the electric dipole. As the plasma frequency approaches the wave frequency, $X_{A}$ beco- mes very large. Unless it is possible to compensate for this by circuit changes, the ratio $V_{\mathbf{L}}^{2} / \mathrm{V}_{\mathbf{A}}^{2}$ will eventually vary in proportion to $n^{4}$. Then $\mathrm{V}_{\mathrm{L}}^{2}$ will be proportional to $n^{5}$ which is a factor $n^{2}$ poorer than the magnetic dipole. This is usually only important for plasma frequencies very close to the wave frequency.

When the plasma frequency exceeds the wave frequency, propagating waves are not supported by the plasma. The radiation resistance of any antenna therefore disappears. The reactance of the electric dipole changes sign and is positive.

\section{Cold plasma with magnetic field.}

The plasma acts as an anisotropic dielectric. It is doubly refracting with refractive indices given by the Appleton-Hartree formula. The behavior of the indices has been discussed by, for example, RATouIFFe [3]. Labels I and II will be applied to the two characteristics waves. Label I will apply to that characteristic wave which is independent of the strength of the magnetic field when propagating perpendicularly to it ; this is widely called the ordinary wave.

It is useful to refer to the $\mathrm{X}-\mathrm{Y}^{2}$ plane (fig. 2) which is divided according to the properties of the Appleton-Hartree formula. Both characteristic waves can propagate in regions $1,3,6$ and 7 . In regions 2 and 8 only wave $I$ can propagate and in region 4 only wave II can propagate. Neither wave can propagate in region 5 so radiation resistance is zero and the impedance of any antenna is purely reactive.

In the cases of wave II in region 3 and wave $I$ in regions 7 and 8 , the refractive index becomes infinite for certain directions relative to the magnetic field. The corresponding phase and group velocities are zero and the wavelength also is zero. These facts give rise to theoretical difficulties which do not yet appear to have been overcome. For example, since the wavelength for certain directions is zero, a real antenna cannot be regarded as electrically small (nor even as thin). In computing the fields at distances great compared with the antenna dimensions, the phase differences between contributions from different parts of the antenna cannot be neglected. This simplification, which may be valid outside regions 3,7 and 8 , is implicit if results derived for a point, or delta function, source are applied to a real antenna. Furthermore, the simple current distribution of an antenna which is electrically small in free space may be expected to change when the antenna is in regions 
3, 7 and 8. As well as complicating impedance theory, this will change the effective length of the antenna. Even though this is the result of the singularity in the refractive index of one characteristic wave, it also introduces difficulties in interpreting observations of the other. Thus, while extraterrestrial radio signals in characteristic wave I may be observed in region 3 of the topside ionosphere, the unknown antenna current distribution caused by the properties of wave II prevents the interpretation of the observations either in terms of radiation resistance or effective length.

There may be a more fundamental objection to most theoretical work that has been done on this topic. In calculating the fields of an antenna excited by a time-harmonic source, it is common to assume steady-state sinusoidal time variation of all field quantities. It may be in fact that such a steady state does not exist, but that energy must be supplied continuously to growing fields. This effect is well-known for an inhomogeneous plasma [4], [5]. Before discussing an antenna in a homogeneous plasma, consider that if an exciting source is applied to a purely reactive impedance, then during the initial transient the impedance absorbs a certain amount of energy. This energy is retained, even though in the subsequent steady state the net flow of energy to the impedance is zero. If now the antenna is in a plasma which has zero group velocity for certain directions, it is possible that infinite time is required to supply all the energy that would be required to achieve a steady state, even though this energy is supplied to the near field which one expects to be essentially reactive. This behavior might be described as an " extended transient ", and may result in an apparently resistive impedance even though reactive fields are being built up.

This suggested behavior should not be confused with some of the results of the works cited below which are all for true steady-state conditions While there is some theoretical interest in this peculiar behavior, the collisionless Appleton-Hartree approximation is not adequate to describe a real plasma. If any loss, however small, is present, or if the kinetic pressure and compressibility of the plasma is taken into account [6], the infinities are suppressed. Moreover, the Appleton-Hartree formula is based on small-signal theory and is inadequate to represent a real plasma in regions of strong fields, associated with the singularities in the refractive index [7].

Outside regions 3,7 and 8 , use of the collisionless Appleton-Hartree approximation appears to give reasonably adequate theories for electrically small antennas. These theories have proved valuable for interpreting radio astronomy observations made in the topside ionosphere.

\subsection{Raliation resistance.}

KOGELNIK [8] has given an expression for radiation resistance of an elementary electric dipole. KoghLNIK and Motz [9] have given a corresponding expression for a small magnetic dipole. These expressions are in the form of intractable integrals. They have been examined by $W_{\text {HIII }}$ and WALSH [10], [11] who give extensive numerical results. The radiation resistance may be broken into two additive components $R_{I}$ and $R_{\text {II. Each }}$ is associated purely with the corresponding characteristic wave. Thus $R_{I}$ vanishes in regions 4 and 5 of figure 2, while $R_{\text {II }}$ vanishes in regions 2, 5 and 8. Each has its own effective temperature associated with it ; in general these temperatures are independent.

Relative to its free space value the total radiation resistance for either an electric or a magnetic dipole may be written

$$
\mathrm{R}_{\mathrm{A}} / \mathrm{R}_{\mathrm{A} 0}=\mathrm{M}+\mathrm{N} \cos 2 \psi,
$$

where $\psi$ is the angle between the magnetic field and the dipole while $M$ and $N$ are functions of $X$ and $Y$. Expressions similar to (3.1) may be written separately for $R_{I}$ and $R_{I I}$. In free space, $M$ is, of course, unity and $N$, which measures the dependence of $R_{A}$ on orientation of the dipole, is zero.

One of the most interesting results of the numerical studies is the behavior of $R_{A}$ for an electric dipole near the boundary between regions 1 and 2. Suppose a path is traced out on figure 2 starting near the origin in region 1 and crossing into region 2. It is found that $M$ decreases slowly at first while $\mathbf{N}$ remains very small and may in fact be negative. As the boundary $\mathrm{X}=1-\mathrm{Y}$ is approached, $M$ begins to decrease very rapidly and $N$ begins to increase very rapidly. On crossing the boundary there are discontinuities in these rates of change to low values in region 2 . These low rates of change continue to the boundary of region 3 where the theory ceases to be valid. The behavior on crossing the boundary from region 1 to 2 is associated with the disappearance of $R_{\text {II. }}$. This behavior has proved to be a useful experimental indicator of the condition $X=1-Y$, and hence permitted accurate determination of electron density in the topside ionosphere. While $R_{I I}$ also disappears in region 2 for a magnetic dipole, the behavior at the boundary is not so abrupt. 
The rates of change of $M$ and $N$ are continuous across the boundary, thus the magnetic dipole is not so useful an indicator of the condition $\mathrm{X}=\mathbf{1}-\mathrm{Y}$.

The work of WALSH, HADDOCK and ScHULTE [12] provides good experimental verification of much of this theory. They observed cosmic noise in the topside ionosphere with a short electric dipole on a spinning rocket. The average signal received during a spin cycle was shown to be in accord with the predictions of Kogelnik's theory over a considerable range of altitude including regions 1 and 2 at 1.225 Mc. In particular, quantitative agreement of the average signal with prediction was reported during the crossing of the boundary $\mathrm{X}=1-\mathrm{Y}$. However, while spin-modulation effects were noted during the boundary crossing they were not investigated quantitatively.

It can now be reported that further investigations show the predictions of the theory agree accurately with the observed changes of phase and amplitude of spin-modulation during the boundary crossing. In region 1, prior to the boundary crossing, modulation of $\mathrm{V}_{\mathrm{L}}^{2}$ was observed with constant amplitude and phase. The depth of modulation was $\pm 15 \%$ of the average value at $1.225 \mathrm{Mc}$, and was apparently due to variation of antenna temperature over the spin cycle. There were marked changes in the phase and less marked changes in the amplitude of modulation in the period around the border-crossing. These were presumed due to the onset of spin-modulation of $R_{A}$ as the coefficient $N$ in (3.1) increased. Using the model ionosphere derived previously and the known geometry of the vehicle motion relative to the local geomagnetic field, the resulting theoretical variation of $R_{A}$ has now been calculated. Assuming the modulation of $T_{\mathbf{A}}$ to remain constant over the period of interest, and taking account of the measured $\mathbf{X}_{\mathbf{A}}$, the predicted modulation of $V_{L}^{2}$ may therefore be determined. It has been found to be in detailed agreement in amplitude and phase with the observations over a period of $200 \mathrm{~s}$ (corresponding to an altitude range of nearly $500 \mathrm{~km}$ ) including the boundary crossing.

Finally, it may be remarked that KogelnIK's formula gives infinite values for $R_{I I}$ in region 3 and for $R_{I}$ in regions 7 and 8 of figure 2. These are associated with the singularities in the refractive indices of the corresponding characteristic waves. However, for the reasons mentioned previously, the theory is of dubious validity in these regions.

\subsection{Reactance.}

KAISER [13] derived an approximate expression for the reactance of a biconical antenna. He explicitly assumed the antenna was so short that the radiation resistance could be ignored and its input impedance evaluated by treating it as a capacity with an anisotropic dielectric. In addition, he employed a certain mathematical procedure with an accuracy difficult to assess. Pyati and WEL [14] have obtained an exact mathematical solution to the same physically simplified problem. Their result is expressed in elliptic integrals. BALMAIN [15], [16] has obtained an approximate solution for the case of a cylindrical antenna. $\mathrm{He}$ examines the fields in much greater detail than the other authors.

All these authors obtain quasi-static solutions. KaIser [13] and Pyati and WeIL [14] solve what may be regarded as a modified Laplace's equation, subject to the boundary conditions that the biconical surfaces are equipotentials with a potential difference equal to the applied voltage. Pyati and WEIL find that KAISER's solution is mathematically exact for a bicone aligned with the static magnetic field. However, for a bicone perpendicular to the magnetic field, KAISER may be appreciably in error ; this error is typically of the order of $20 \%$. Balmain [15], [16] solves a modified Poisson's equation, assuming a suitable charge distribution. He primarily studies uniform charge distribution on each half of the antenna, corresponding to a triangular current distribution, but also considers other distributions.

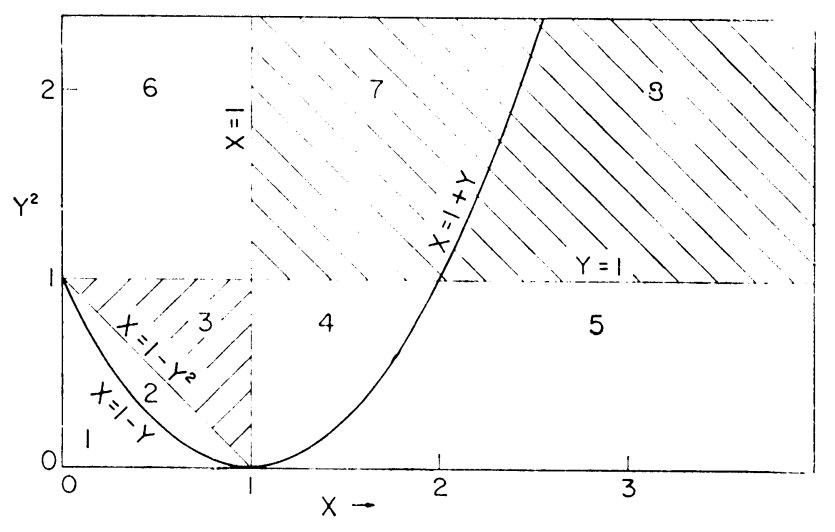

Frg. 2. - The X - Y 2 plane.

These solutions all have the same basic characteristics, and differ only by relatively small factors. Referring to figure 2 , the reactance $\mathrm{X}_{\mathbf{A}}=\mathbf{X}_{\mathbf{A}_{0}} /(\mathbf{l}-\mathbf{X})$ on the line $\mathbf{Y}=0$, as would 
be expected. Also, $\mathbf{X}_{\mathbf{A}}=\infty$ on the lines $\mathrm{X}=1-\mathrm{Y}^{2}$ and $\mathrm{X}=1$, and $\mathrm{X}_{\mathrm{A}}=0$ on the line $Y=1$. In regions 1,2 and $6, X_{A}$ is negative (capacitive), and in regions 4 and 5 it is positive (inductive).

The fields studied have $r^{3}$ dependence for sufficiently large values of $r$, so may reasonably be called near fields. However, in regions 3,7 and 8 , BatumaIN [15], [16] finds singular behavior of the fields. He shows that it is only beyond a transition region that $r^{-3}$ behavior occurs, and the distance of this transition region is inversely proportional to collision frequency. For the collisionless case, the " near field " therefore extends to infinite distance. This behavior is related to the fact that in regions 3,7 and 8 in the collisionless case, the impedance associated with these field components is found to be complex ; it contains a resistive as well as reactive component. This latter effect is found also by KaIser [13] and by Pyati and WEIL [14]. For the reasons discussed earlier, the results of these theoretical studies are of doubtful physical significance.

There appears to be little precise experimental verification of these theoretical predictions. BALMaIN [15], [16] has made laboratory measurements under conditions of fairly high collision frequency. He finds fairly good qualitative agreement with the predictions of his theory. WALSH, HADDOCK and Schulte [12] made measurements in the topside ionosphere under conditions of very low collision frequency. They found good agreement with a slightly modified version of KAISER's formula in regions 1 and 2 of figure 2 .

\section{WARM PLASMA WITHOUT MAGNETIC FIELD.}

When electron pressure due to thermal motions is taken into account, then longitudinal compressional waves may exist. The excitation of these by an antenna immersed in the plasma has been the subject of much discussion, but no realistic theory seems to exist.

In a series of three papers, CoHen [17], [18], [19] has discussed the fields and waves that can exist in a plasma and their relation to exciting sources. $\mathrm{He}$ treats a homogeneous, neutral plasma as a continuous fluid with no superposed magnetic field, and examines the linearized macroscopic equations governing small perturbations. The fields may be divided into two uncoupled groups which Cohen refers to as the electromagnetic (EM) and the plasma (P) components. The EM component includes all of the magnetic field and no charge accumulation and may be excited by both currents and charges. It is just the field that would exist if the plasma were cold. As far as the EM field is concerned, the plasma behaves exactly as a dielectric medium with dielectric constant $1-\mathrm{X}$. The $\mathrm{P}$ component has no magnetic field and all the charge accumulation. It may be excited by charges but not by currents. At great distances from the source, it becomes a longitudinal (electroacoustic) plasma wave with the dispersion relation of BoHM and Gross [20]. The P field does not exist in a cold plasma. If the plasma is inhomogeneous, as for example at a boundary, then coupling between the two types of field may occur. If the r. m. s. thermal velocity of electrons is $v_{0}$, then the wavelength of plane $P$ waves of frequency $f$ is $\lambda_{p}=v_{0} / f(1-\mathrm{X})^{1 / 2}$. In the topside ionosphere, $v_{0}$ is typically $200 \mathrm{~km} / \mathrm{s}$ so $\lambda_{p}=20$ .$(1-\mathrm{X})^{-1 / 2} \mathrm{~cm}$ at $f=1 \mathrm{Mc}$. Thus, the antennas used for many applications in space radio astronomy have diameters $\ll \lambda_{p}$ but lengths $\gg$ $\lambda_{p}$, even though they are short compared with an electromagnetic wavelength.

CoHen [19] approximated a linear dipole by a filament of current in a homogeneous plasma. $\mathrm{He}$ calculated the radiation resistance $R_{p}$ due to energy radiated in $P$ waves by integrating the power crossing a sphere of very large radius. Unfortunately, he chose a current distribution which was certainly incorrect. If $P$ waves are launched by an antenna, they will presumably affect the current distribution. The calculation of the correct distribution is difficult. However, assuming as a first approximation the simple triangular current distribution of a dipole electrically short in free space, CoHEN's procedure may be carried out to obtain the result, for an antenna of total length $\mathrm{L} \gg \lambda_{p}$,

$$
\mathrm{R}_{p}=60 \frac{\lambda_{0}}{\mathrm{~L}} \frac{\mathrm{X}}{1-\mathrm{X}} \text { ohm. }
$$

$\lambda_{0}$ is the free space electromagnetic wavelength for the frequency of interest. This resistance is very large compared with the electromagnetic radiation resistance in many cases and increases rapidly as $X$ approaches unity. The problem of loading by $\mathbf{P}$ waves is thus of great practical significance.

BaLMarN [15] has attempted to take into account the influence of antenna diameter by considering the current to be distributed on a hollow, thin cylindrical surface in a homogeneous plasma. He uses the " reaction concept", calculating in essence the complex work done by the current distri- 
bution on the $\mathrm{P}$ component of electric field at the antenna surface. This technique also gives an expression for the reactance $\mathbf{X}_{p}$ associated with the $\mathrm{P}$ field. For total length $\mathrm{L} \gg \lambda_{\mathrm{p}}$ and an assumed triangular current distribution, BALMAIN's expression for $R_{p}$ reduces to (4.1) multiplied by a factor $\mathrm{J}_{0}^{2}\left(k_{\mathrm{p}} a\right)$. Here $a$ is the radius of the cylinder, $k_{\mathrm{p}}=2 \pi / \lambda_{v}$, and the usual notation for the Bessel function of the first kind is used. For many cases of interest in the topside ionosphere, this correction factor is not greatly different from unity. The expression for $\mathrm{X}_{p}$ is equal to (4.1) multiplied by a factor $-\mathrm{J}_{0}\left(k_{\mathrm{p}} a\right) \mathrm{N}_{0}\left(k_{p} a\right)$, the usual notation for the Bessel function of the second kind being used. This is a very sensitive function of radius, being zero at $a=0.142 \lambda_{p}$, positive (inductive) for smaller values of $a$, and rising to $\infty$ as $a$ goes to zero.

The analyses of CoHEN and BaLmaIn are highly idealized in that they assume a homogeneous plasma with a current distribution unsupported by metallic boundaries. CoHEN [19] recognizes that the presence of metal boundaries may alter the results appreciably. The most important consequence is the appearance of inhomogeneities in the plasma around the antenna. This normally takes the well-known form of a sheath deficient of electrons. The electron density may be almost zero close to the metallic wall, rising gradually to its unperturbed vaiue in the neutral plasma at a distance of several Debye lengths. The Debye length is typically $2 \mathrm{~cm}$ in the topside ionosphere, so the dimension of the region around the antenna which is appreciably perturbed may be comparable to $\lambda_{p}$. The absence of electrons close to the metallic surface means that, as far as radio frequency effects are concerned, this region acts as a vacuum. The fields in this region are then essentially of the EM type. If $\mathrm{P}$ fields are to be produced in the homogeneous region outside the sheath, it is necessary to calculate the coupling between these fields through the transition region of the sheath. It is not possible to identify two uncoupled groups of fields in this region.

One approach to this problem [21] has attempted to take the sheath into consideration by treating it as a discontinuous, rigid boundary between an electron-free region and the unperturbed plasma. This is certainly an artificial model, and amounts to an arbitrary assumption of the amplitude of the $P$ field outside the boundary. Thus, a quasi-electrostatic approach is taken and the calculation implicitly proceeds as follows. The EM field both inside and outside the boundary has only an elec- tric component. It is normal to both the metallic surface and the plasma boundary, and is the same as if the plasma were cold. It may be calculated in a straightforward manner, treating the plasma as a medium with dielectric constant $1-\mathrm{X}$, assuming that somehow a boundary is maintained which is discontinuous but not rigid, so that oscillations of the electrons at the boundary may take place normal to the boundary and the necessary boundary conditions on the EM field may be satisfied. It is then assumed that the oscillations of these electrons due to the $P$ field, which exist only in the plasma, are of equal amplitude but in antiphase to those of the EM field. Thus, the electrons at the boundary are permanently at rest, satisfying the assumption of rigidity, and no surface charge appears. The electrical boundary condition on the $\mathbf{P}$ field is automatically satisfied, as discussed later. There seems to be no physical basis for the assumption of equal amplitude EM and $P$ oscillations of electrons at the boundary.

WHALE [22] has treated a linear antenna surrounded by a cylindrical sheath which he assumes to have a discontinuous boundary. He does not explicitly assume a rigid boundary though he appears to suggest the amplitude of electron oscillation due to the $\mathbf{P}$ field at the boundary is comparable to that due to the EM field. He assumes all fields to be strictly radial so that the $P$ field is an expanding cylindrical wave. His derivation of the amplitude of the $\mathrm{P}$ wave is somewhat obscure. He apparently assumes the $\mathrm{P}$ and EM fields interact continuously throughout the plasma, as opposed to the linear separation in a homogeneous plasma deduced by CoHEN [17]. (The important equations 1,2 and 5 in WHALE's appendix seem to be dimensionally incorrect and do not lead to his equation 6). Nevertheless, the concept of an expanding cylindrical wave close to the antenna is interesting. It seems worthwhile to explore it further using CoHEN's separated equations.

Consider an infinitely long filament of charge along the $z$-axis, with uniform charge per unit length $\sigma=\sigma_{0} \exp (-i \omega t)$, that is, a volume charge density $\sigma \delta(x) \delta(y)$. This distribution strictly cannot exist, but it should lead to nearly correct results for the fields at sufficiently small radial distance from, and not too near the ends of, a thin dipole. The EM component of electric field at small radial distance $r$ is then

$$
\mathrm{E}_{e}=\sigma\left[2 \pi \varepsilon_{0}(1-\mathrm{X}) r\right]^{-1},
$$

i. e., the quasi-static field for a cold plasma.

The perturbation $n_{1}$ in the electron density asso- 
ciated with the $P$ field satisfies the inhomogeneous wave equation,

$$
\left(\nabla^{2}+k_{p}^{2}\right) n_{1}=-\left(\sigma \omega_{p}^{2} / e v_{0}^{2}\right) \delta(x) \delta(y)
$$

where $\omega_{p}$ is the radian plasma frequency and $-e$ the charge on the electron. Setting $\partial / \partial z=\partial / \partial \Phi=0$, the solution of this equation is an expanding cylindrical wave, expressed by a Hankel function,

$$
n_{1}=i\left(\sigma \omega_{p}^{2} / e v_{0}^{2}\right) \mathrm{H}_{0}^{(1)}\left(k_{p} r\right) .
$$

The associated radial electric field is

$$
\mathrm{E}_{p}=-i\left(\sigma \omega_{p}^{2} / 4 \varepsilon_{0} k_{p} v_{0}^{2}\right) \mathrm{H}_{1}^{(1)}\left(k_{p} r\right)
$$

The radial velocity of oscillation of an electron is

$$
\text { (4.6) } \quad v_{\mathfrak{p}}=-\left(\sigma \omega e / 4 \varepsilon_{0} m k_{p} v_{0}^{2}\right) \mathrm{H}_{1}^{(1)}\left(k_{p} r\right) \text {, }
$$

where $m$ is the electron mass. The average radial rate of energy flow per unit area is $\frac{1}{2} \operatorname{Re}\left(m v_{0}^{2} n_{1} v_{p}^{*}\right)$. This quantity is readily shown to vary as $r^{-1}$. Integrating this over the surface of a cylinder of unit height and radius $r$, the result is then independent of $r$, and may be interpreted as the rate of loss of energy in $P$ waves per unit length of the radiating source. This quantity is

$$
\mathrm{W}=\frac{\omega \sigma_{0}^{2}}{8 \varepsilon_{0}} \frac{\mathrm{X}}{\mathrm{l}-\mathrm{X}}
$$

It will now be assumed that this formula may be applied to a real current distribution of finite length, $\mathrm{I}=\mathrm{I}_{0} \exp (-i \omega t), \mathrm{I}_{0}$ being a function of $\mathrm{z}$. By continuity, $\sigma_{0}^{2}=\omega^{-2}\left(\partial \mathrm{I}_{0} / \partial z\right)^{2}$. Integrating (4.7) along the length of the antenna yields the total radiated power in $P$ waves ; setting this equal to $\left(I_{m}^{2} / 2\right) R_{p}$, where $I_{m}$ is the value of $I_{0}$ at the driving point, yields the radiation resistance,

$$
\mathrm{R}_{p}=15 \lambda_{0} \frac{\mathrm{X}}{1-\mathrm{X}} \frac{1}{\mathrm{I}_{m}^{2}} \int\left(\frac{\partial \mathrm{I}_{0}}{\partial \mathrm{z}}\right)^{2} \mathrm{dz} \text { ohm }
$$

As a check on this equation, consider the case of a triangular current distribution, $\left(\partial \mathrm{I}_{0} / \partial z\right)^{2}=\left(2 \mathrm{I}_{m} / \mathrm{L}\right)^{2}$; inserting this in (4.8) yields the result (4.1). For a homogeneous plasma, it seems reasonable to apply (4.8) provided I does not vary greatly over a distance $\lambda_{p} . \mathrm{R}_{p}$ is a function of the unknown current distribution; however, it may be shown that the current distribution leading to the minimum value of (4.8) is in fact the simple triangular one. Thus (4.1) represents the minimum value of $R_{p}$ for any current distribution.

If now the current filament is surrounded by a cylindrical sheath, the $\mathrm{P}$ field outside the sheath obeys the homogeneous wave equation obtained by setting the right side of (4.3) equal to zero. Again the solution is expressed in Hankel functions, like (4.4), (4.5) and (4.6), but with an unknown amplitude factor determined by the conditions of excitation. As a first attempt at determining this factor, it might be worthwhile to suppose that a discontinuous boundary be maintained somehow, but without the restriction of rigidity. The results in this case will therefore be discussed next.

Inside the sheath, the EM component of the radial electric field is $\mathrm{E}_{e 1}=\sigma /\left(2 \pi \varepsilon_{0} r\right)$; outside the sheath it is $\mathrm{E}_{e_{2}}=\sigma / 2 \pi \varepsilon_{0} r(1-\mathrm{X})$. A surface charge distribution $\rho^{e}=\varepsilon_{0}\left(E_{2}-E_{1}\right)$, due to motion of electrons in the boundary layer, accounts for the discontinuity in $\mathrm{E}$. This is the normal behavior at the surface of a medium with dielectric constant $1-X$. Suppose in addition $a$ cylindrical $\mathrm{P}$ wave exists outside the sheath with radial electric field $\mathrm{E}_{p}$. Then the velocity of electrons associated with this electric field and the perturbation volume charge density are defined. The associated surface charge density $p_{D}$ due to motion of electrons in the boundary layer may therefore be calculated, and is $\varepsilon_{0} \mathrm{E}_{p}$. That is to say, the surface charge density associated with the $\mathrm{P}$ wave is exactly that required to support a discontinuity $\mathrm{E}_{p}$. The $\mathrm{P}$ wave is " self-contained ". No relationship to the electric field inside the sheath is obtained. A P wave of any amplitude can exist and satisfy the electrical boundary conditions. The problem of finding the amplitude of an excited $P$ wave is therefore indeterminate.

The reason for this indeterminacy is that in addition to electrical boundary conditions, mechanical ones must be satisfied. These are continuity of electron pressure and velocity. The assumed discontinuous sheath model is incapable of satisfying these. The problem can of course be made determinate, as mentioned previously, by the quite artificial assumption of a rigid boundary.

It appears that in order to solve this problem, the continuous nature of the sheath must be taken into account. Simplified approaches must be treated with caution. Ideally a solution of the radio frequency fields should be obtained using full equations without linearization. A macroscopic, continuous fluid approach may in fact be inadequate, since the distribution of electron kinetic velocities in the sheath is anisotropic and nonMaxwellian.

It may well be that a sheath acts very efficiently to decouple an antenna from $\mathbf{P}$ waves. It is inte- 
resting to note an observation of $R$. F. effects in a laboratory plasma sheath by HARP [23]. The plasma was excited by frequencies close to the plasma frequency and an electron beam probe technique of diagnosis was used. A P wave should have been readily detectable, but in fact no trace could be found, even when special efforts were made. There is a deartb of experimental work on $P$ wave effects on antennas and more experimental work would be of great value.

Manuscrit reçu le 23 mars 1965.

\section{REFERENCES}

[1] HuanL J., 1961, Planet. Space Sci., 8, 68.

[2] Budden K. G. and Huarll J., 1964, Proc. Roy. Soc. $A, 277,365$.

[3] Ratoumre J., 1959, Magneto ionic Theory (Cam. bridge University Press).

[4] Aluis W. P., Buorsbaum S. J. and Bers A., 1963, Waves in Anisotropic Plasmas (M. I. T. Press, Cambridge, Mass.), 13-15.

[5] BUdDen K. G., 1961, Radio Waves in the Ionosphere (Cambridge University Press), 479.

[6] Référence [4], Ch. 5.

[7] Arbel E. and Frusen L. B., 1963, Electromagnetic Theory and Antennas, Part I (Pergamon Press), 421.

[8] KogkLNIK H., 1960, J. Res. NBS, 64D, 515.

[9] KoGRLNIK H. and Motz H., 1963, Electromagnetic Theory and Antennas, Part I (Pergamon Press), 477.

[10] Wrir H. and WaLsh D., 1964, Trans. IEEE, $A P-12,297$.

[11] WeII H. and WALSH D., 1965, Trans. IEEE, $A P-13,21$.
[12] Walsh D., Haddock F. T. and Sohulte H. F., 1964, Space Research, IV (North-Holland Publ. Amsterdam), 935.

[13] KaIser T. R., 1962, Planet. Space Sci., 9, 639.

[14] Pyati V. P. and WeIL H., 1965, Radio Sci. $J$. Res. NBS/USNC.URSI, 69D, 291.

[15] Balmatn K. G., 1964, Aeronomy Report No 2, Univers ty of Illinois, Urbana, Illinois.

[16] Balmain K. G., 1964, Trans. IEEE, AP-12, 605.

[17] Cohen M. H., 1961, Phys. Rev., 123, 711.

[18] Cohen M. H., 1962, Phys. Rev., 126, 389.

[19] Cohrw M. H., 1962, Phys. Rev., 126, 398.

[20] Вонм D. and Gross E. P., 1949, Phys. Rev., 75, 1851.

[21] Fruer J. A., 1964, Radio Sci. J. Res. NBS/USNCURSI, 68D, 1171.

[22] Whale H. A., 1963, J. Geophys. Res., 68, 415.

[23] HaRp R. S., 1964, M. L. Report No. 1175 (Microwave Laboratory, Stanford University, Stanford, California), 60. 